PREPARED FOR THE U.S. DEPARTMENT OF ENERGY, UNDER CONTRACT DE-AC02-76CH03073

PPPL-3908

PPPL-3908

UC-70

Aspect Ratio Scaling of Ideal No-wall Stability Limits in High Bootstrap Fraction Tokamak Plasmas

by

J.E. Menard, M.G. Bell, R.E. Bell, D.A. Gates, S.M. Kaye, B.P. LeBlanc, R. Maingi, S.A. Sabbagh, V. Soukhanovskii, D. Stutman, and the NSTX National Research Team

November 2003
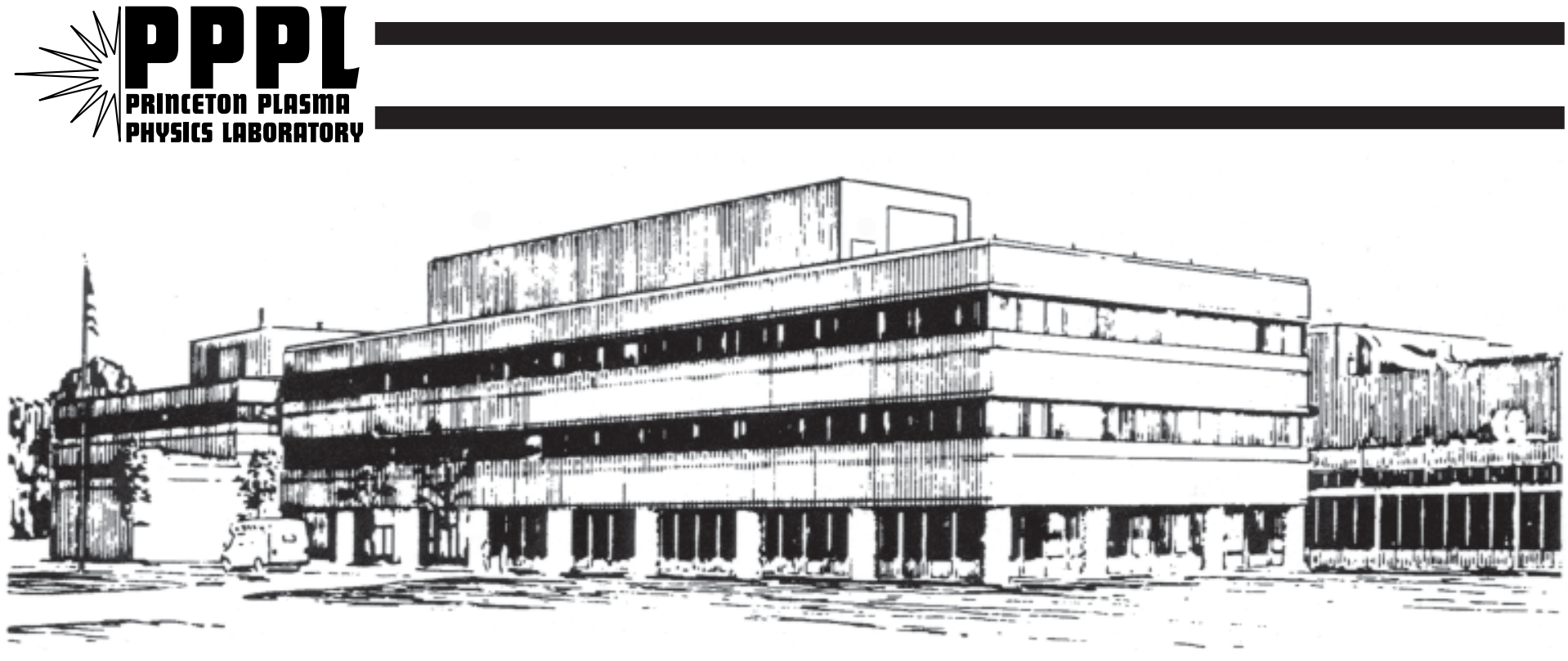

PRINCETON PLASMA PHYSICS LABORATORY PRINCETON UNIVERSITY, PRINCETON, NEW JERSEY 


\section{PPPL Reports Disclaimer}

This report was prepared as an account of work sponsored by an agency of the United States Government. Neither the United States Government nor any agency thereof, nor any of their employees, makes any warranty, express or implied, or assumes any legal liability or responsibility for the accuracy, completeness, or usefulness of any information, apparatus, product, or process disclosed, or represents that its use would not infringe privately owned rights. Reference herein to any specific commercial product, process, or service by trade name, trademark, manufacturer, or otherwise, does not necessarily constitute or imply its endorsement, recommendation, or favoring by the United States Government or any agency thereof. The views and opinions of authors expressed herein do not necessarily state or reflect those of the United States Government or any agency thereof.

\section{Availability}

This report is posted on the U.S. Department of Energy's Princeton Plasma Physics Laboratory Publications and Reports web site in Fiscal Year 2004. The home page for PPPL Reports and Publications is: http://www.pppl.gov/pub_report/

DOE and DOE Contractors can obtain copies of this report from:

U.S. Department of Energy

Office of Scientific and Technical Information

DOE Technical Information Services (DTIS)

P.O. Box 62

Oak Ridge, TN 37831

Telephone: (865) 576-8401

Fax: (865) 576-5728

Email: reports@adonis.osti.gov

This report is available to the general public from:

National Technical Information Service

U.S. Department of Commerce

5285 Port Royal Road

Springfield, VA 22161

Telephone: $1-800-553-6847$ or

(703) $605-6000$

Fax: (703) 321-8547

Internet: http://www.ntis.gov/ordering.htm 


\title{
Aspect ratio scaling of ideal no-wall stability limits in high bootstrap fraction tokamak plasmas
}

\author{
J.E. Menard ${ }^{1}$, M.G. Bell ${ }^{1}$, R.E. Bell ${ }^{1}$, D.A. Gates ${ }^{1}$, S.M. Kaye ${ }^{1}$, B.P. LeBlanc ${ }^{1}$, R. Maingi ${ }^{2}$, \\ S.A. Sabbagh ${ }^{3}$, V. Soukhanovskii ${ }^{1}$, D. Stutman ${ }^{4}$, and the NSTX National Research Team. \\ ${ }^{1}$ Princeton Plasma Physics Laboratory, \\ Princeton, NJ \\ ${ }^{2}$ Oak Ridge National Laboratory, \\ Oak Ridge, TN \\ ${ }^{3}$ Columbia University, New York, NY \\ ${ }^{4}$ Johns Hopkins University, Baltimore, $M D$
}

(Dated: November 20, 2003)

\begin{abstract}
Recent experiments in the low aspect ratio National Spherical Torus Experiment (NSTX) [M. Ono et al., Nucl. Fus. 40, 557 (2000)] have achieved normalized beta values twice the conventional tokamak limit at low internal inductance and with significant bootstrap current. These experimental results have motivated a computational re-examination of the plasma aspect ratio dependence of ideal no-wall magnetohydrodynamic stability limits. These calculations find that the profileoptimized no-wall stability limit in high bootstrap fraction regimes is well described by a nearly aspect ratio invariant normalized beta parameter utilizing the total magnetic field energy density inside the plasma. However, the scaling of normalized beta with internal inductance is found to be strongly aspect ratio dependent at sufficiently low aspect ratio. These calculations and detailed stability analyses of experimental equilibria indicate that the non-rotating plasma no-wall stability limit has been exceeded by as much as $30 \%$ in NSTX in a high bootstrap fraction regime.
\end{abstract}

PACS numbers: 52.55.Fa, 28.52.Av 


\section{INTRODUCTION}

The superconducting advanced tokamak [1-5] is presently the leading candidate for producing an efficient magnetic fusion reactor. Alternative concepts such as the compact stellarator $[6,7]$ and spherical torus [8-10] are also being actively pursued as possible improvements to the advanced tokamak. The advanced tokamak (AT) and spherical torus (ST) reactor concepts have several features in common. In particular, both rely on the neoclassical bootstrap current [11] to sustain nearly all of the plasma current and on stabilization of pressure-driven external kink modes to achieve sufficiently high beta (ratio of plasma kinetic pressure to magnetic pressure) to produce power efficiently. For these wall-stabilized and fully bootstrap-sustained scenarios, very broad pressure and current profiles with elevated magnetic safety factor in the plasma core are found to optimize ideal magnetohydrodynamic (MHD) stability. Attempting to achieve these plasma configurations in near steady-state conditions is a major challenge of present AT and ST research.

An important element in determining the success in achieving these advanced configurations discussed above is an accurate calculation of the no-wall and ideal-wall stability limits. While stability analysis using magnetic reconstruction and measured internal profiles is required to determine the proximity of a particular discharge to computed stability limits to high accuracy, several useful scalings exist to guide this determination for standard aspect ratio tokamaks. It is well established both theoretically [12-15] and experimentally [16-18] that the maximum stable toroidal beta $\beta_{T} \equiv 2 \mu_{0}\langle p\rangle / B_{T 0}^{2}$ in a tokamak scales as the normalized current $I_{N} \equiv I_{P} / a B_{T 0}$. Here $\langle p\rangle$ is the volume-average plasma pressure, $B_{T 0}$ is the vacuum toroidal field at the plasma geometric center $R_{0}, I_{P}$ is the plasma current, $a$ is the plasma minor radius, and the plasma aspect ratio $A \equiv R_{0} / a$. The leading order scaling of beta with normalized current is commonly removed when comparing different plasma configurations by defining a normalized beta $\beta_{N} \equiv \beta_{T} a B_{T 0} / I_{P}$ with units $\% m T / M A$. The maximum MHD-stable $\beta_{N}$ is then commonly studied as a function of plasma profiles, boundary shape, and conducting wall location. The no-wall normalized beta limit has been empirically found to be roughly proportional to the dimensionless current profile internal inductance $l_{i}[19]$. 
Here $l_{i} \equiv\left\langle B_{P}^{2}\right\rangle /{\overline{B_{P}}}^{2}$ where \langle\rangle again denotes volume average, $\overline{B_{P}}=\mu_{0} I_{P} / l_{P}$, and $l_{P}$ is the poloidal arc-length of the plasma boundary. For standard aspect ratio tokamaks, generally the proportionality factor $\lambda \equiv \beta_{N} / l_{i} \leq 4$ in the absence of wall stabilization. In contrast, theouretical wall-stabilized reactor optimizations often result in plasma configurations with $\lambda=10-20$ for the AT and $\lambda=20-40$ for the ST. Clearly, determining the experimental dependence of the wall-stabilized beta limits on current profile will be very important for both the AT and ST reactor concepts.

In standard aspect ratio tokamaks, significant experimental progress has been made toward achieving sustained high self-driven current fraction [20-22] and in sustaining operation above the no-wall stability limit [23] for external kink modes by utilizing a nearby conducting wall, plasma rotation, and internal dissipation to stabilize the resistive wall mode. Previous experiments on the low-aspect-ratio Small Tight Aspect Ratio Tokamak (START) device [24-27] achieved very high values of toroidal beta approaching $40 \%$ with $\beta_{N} \leq 6$ and $\beta_{N} / l_{i} \leq 6$ but with comparatively higher collisionality and low bootstrap fraction. Only recently has performance approaching that obtained in standard tokamaks become possible in high temperature mega-ampere class spherical torus plasmas in the Mega-Ampere Spherical Tokamak (MAST) [28] and National Spherical Torus Experiment (NSTX) [29] devices. Recent experiments in the NSTX device have largely reproduced START results by achiev$\operatorname{ing} \beta_{T} \leq 35 \%$ and $\beta_{N} \leq 6.5[30,31]$. However, significantly higher $\beta_{N} / l_{i} \leq 10$ has also been achieved in NSTX in high poloidal beta plasmas with bootstrap fractions up to $50 \%$. Unlike other present-day ST experiments, NSTX was constructed with close-fitting passive stabilizing plates to suppress external kink modes, so wall stabilization may account for the ability to achieve high $\beta_{N} / l_{i}$. Another possibility is that conventional aspect ratio scalings for the normalized beta limit become invalid at low aspect ratio. The results from START are consistent with the later explanation since the conducting wall of the device (i.e. its vacuum chamber) was far from the plasma yet the maximum $\beta_{N} / l_{i}$ achieved approached 6 .

The apparent significant variation of normalized stability limits with plasma aspect ratio motivates the present work which seeks to determine if any such scalings like those discussed above are potentially useful in describing beta limits independent of plasma aspect ratio. 
The remainder of this article is organized as follows. Section II describes systematic stability scans varying plasma aspect ratio, shape, and normalized current. Section III compares the results of the these scans to NSTX experimental data. Finally, Section IV summarizes these results.

\section{SYSTEMATIC STABILITY SCANS}

The numerical methods and definitions used here for determining equilibrium and stability are well established and have been described in a previous study of low aspect ratio ideal stability [32]. In addition, the DCON stability code [33] is also now routinely used. As in the previous study above, the plasma boundary shape is specified as an up/down symmetric limiter boundary (no separatrix) defined by

$$
X_{b}(\theta)=R_{0}+a \times \cos (\theta+\delta \sin (\theta))
$$

and

$$
Z_{b}(\theta)=\kappa a \times \sin (\theta)
$$

where $\kappa$ is the elongation and $\delta$ is the triangularity. In the following analysis, ideal beta limits are determined for equilibria which are constrained to have no local bootstrap current overdrive and which are marginally stable to ballooning modes and $n=1-3$ kink modes without wall stabilization. For most cases treated, the optimization of the pressure and current profiles results in the equilibrium being simultaneously marginally stable to ballooning and $n=1$ kink modes. Five free parameters are used in the specification of the pressure profile and seven are used for the parallel current density profile. The normalized pressure is specified as

$$
\hat{p}(\psi)=w \times\left(1-\hat{\psi}^{b_{p 1}}\right)^{a_{p 1}}+(1-w) \times\left(1-\hat{\psi}^{b_{p 2}}\right)^{a_{p 2}}
$$

and the current profile as

$$
\frac{\langle\vec{J} \cdot \vec{B}\rangle}{\langle\vec{B} \cdot \nabla \phi\rangle}(\psi) \propto\left(1-\hat{\psi}^{b_{c 1}}\right)^{a_{c 1}}+C \times \frac{\hat{\psi}^{a_{1}}(1-\hat{\psi})^{a_{2}}}{(\hat{\psi}-A)^{2}+D^{2}}
$$


in the equilibrium solution. Here $\psi$ is the poloidal flux and the normalized poloidal flux $\hat{\psi} \equiv\left(\psi-\psi_{\text {axis }}\right) /\left(\psi_{\text {edge }}-\psi_{\text {axis }}\right)$. The pressure profile is constrained to have zero edge gradient and the current profile to have zero edge current density to avoid edge-localized kink-ballooning modes. The normalized temperature profile has the same functional form as that shown in Equation 3 and the density and temperature profile functions are chosen to have similar peaking factor. A constant $Z_{\text {eff }}=2$ is assumed in the specification of the collisionless bootstrap current density profile, and adjustments to the optimized current profile are made to eliminate any local bootstrap current overdrive. In the results described below, each pressure and current profile parameter was varied iteratively until the stable normalized beta increased no more than $1 \%$ during a profile parameter variation cycle. Such convergence typically required 2 to 4 variation cycles. A total of approximately 4700 equilibrium calculations were used to obtain the stability limit results described below.

\section{A. Plasma aspect ratio scan}

The first systematic scan performed varied the plasma aspect ratio from $A=1.25$ to 10 constraining the self-driven current fraction to be 50-55\%. The plasma boundary shape was fixed with elongation $\kappa=2.0$ and triangularity $\delta=0.45$ - values typical of present-day standard aspect ratio and low aspect ratio tokamak plasmas. With the bootstrap fraction and cross-sectional shape constrained, Figure 1a shows an order of magnitude increase in toroidal beta as the aspect ratio is decreased from 10 to 1.25. This increase is larger than that expected from aspect ratio variation alone, as it can be shown [32] that $\beta_{T} \sim A^{-1 / 2}(1+$ $\left.\kappa^{2}\right) \beta_{N}^{2} / f_{B S}$, implying that the explicit dependence of the toroidal beta limit on aspect ratio is relatively weak when the bootstrap current fraction $f_{B S}$ and elongation are fixed. Thus, the additional factor of three increase in toroidal beta is the result of the near doubling of $\beta_{N}$ as $A$ is decreased as shown in Figure 1b. Importantly, Figure 1b also shows that a normalized beta parameter utilizing the total magnetic field energy density inside the plasma as originally chosen by Troyon [13] is an approximate aspect ratio invariant of stability with a standard deviation of only $3 \%$ and mean value of 3.2 for the geometry and profile constraints 
used here. Here the volume-average normalized beta is defined as $\left\langle\beta_{N}\right\rangle \equiv\langle\beta\rangle_{a} B_{T 0} / I_{P}$ again in $\% m T / M A$ where the volume-average beta $\langle\beta\rangle \equiv 2 \mu_{0}\langle p\rangle /\left\langle B^{2}\right\rangle$. Thus, Figure $1 \mathrm{~b}$ also clearly shows that the degeneracy in possible definitions of normalized beta $[12,13]$ is removed at low aspect ratio by the combined effects of strong toroidicity (i.e. variation of $1 / R$ on a flux surface) and enhanced poloidal field strength relative to the toroidal field. Figure 1c shows that the optimized pressure profiles are relatively broad with pressure peaking factors less than 2.5 with the lowest peaking factors occuring at the lowest aspect ratio treated. Figure $1 \mathrm{~d}$ shows that the optimal central safety factor $q(\hat{\psi}=0)$ is approximately 2 for $A<1.6$ and 1 to 1.3 for $A>2$ with $A=1.8$ an apparent transitional aspect ratio lying between the spherical torus and tokamak. Further, for $A>2$, the optimal $q$ profile was found to have weakly reversed shear with $q(0)-\operatorname{MIN}(q)<0.1$, whereas the lower aspect ratio cases had monotonic $q$ profiles. Interestingly, this figure also shows that the optimal inverse internal inductance is coincidently nearly identically equal to $q(0)$. Figure 1e shows that for $A>2$, the variation of the optimal $\left\langle\beta_{N}\right\rangle / l_{i}$ is small and has a mean value of 3.8 , while $\beta_{N} / l_{i}$ varies from 4.5 to 5.5 as $A$ decreases from 3 to 2 . These findings for $A>2$ are roughly consistent with the emperical scaling $\beta_{N} / l_{i} \leq 4$ discussed above for standard aspect ratio tokamaks. However, Figure 1e also shows that the decrease in $l_{i}$ and increase in optimized $\beta_{N}$ as the aspect ratio is lowered yields $\beta_{N} / l_{i}$ and $\left\langle\beta_{N}\right\rangle / l_{i}$ ratios which are strong functions of aspect ratio for $A<2$. These results clearly indicate that ratios like $\beta_{N} / l_{i}$ cannot be aspect ratio invariants of no-wall ideal stability. However, it is still possible that the no-wall normalized beta limit scales as $l_{i}$ for fixed aspect ratio, but this possibility has not been investigated here.

\section{B. Plasma shape scan}

While Figure $1 \mathrm{~b}$ shows that $\left\langle\beta_{N}\right\rangle$ is nearly aspect ratio invariant for the specfic constraints chosen, numerous previous studies for standard aspect ratio tokamaks $(A \approx 3)$ have shown that normalized beta limits can be significantly influenced by boundary shape and profile constraints [34-38]. For circular cross-section plasmas with reversed-shear $q$ profiles and 
$\operatorname{MIN}(q)=1.2$, no-wall $\beta_{N}$ limits up to 3.5 have been reported [35] at pressure peaking factor $p(0) /\langle p\rangle=3$. These values are similar to those shown in Figure 1 for similar aspect ratio despite the large difference in boundary shape. Another study [36] which systematically varied the pressure and current profiles consistent with good bootstrap current alignment for shaping parameters $\kappa=1.6-2.0$, and $\delta=0.3-0.7$ found a range of combined ballooning and $n=1$ kink limits with $3.3 \leq \beta_{N} \leq 4.3$ and $\beta_{N} / l_{i} \leq 6$. Another similar study [38] performed without pressure profile optimization treated $\kappa=1.8$ and a wider range of triangularity $\delta$ $=0.1-0.7$ and found $n=1$ kink no-wall $\beta_{N}$ limits in the range of 2.5 to 4 with the lowest $\beta_{N}$ limits occuring at the lowest triangularities.

The highest normalized beta limits reported in the above studies (without wall stabilization) appear to be within 10\% of the values shown in Figure 1 for similar internal inductance and pressure profile peaking factors. It is therefore inferred that those equilibria also had $\left\langle\beta_{N}\right\rangle$ upper limits similar to those shown in Figure 1b. Thus, to further test the near aspect ratio invariance of $\left\langle\beta_{N}\right\rangle$ discussed above, a no-wall stability scan at lower aspect ratio $A=1.6$ was performed with varied plasma shape and fixed self-driven current fraction of 50\%. Figure 2a shows that for shape parameters $\kappa=1.6-2.5$ and $\delta=0.3-0.6$ at $A=1.6$, no-wall kink and ballooning marginally stable toroidal beta can vary as much as a factor of 4 depending on shape. For these shape changes, Figure $2 \mathrm{~b}$ shows that the normalized toroidal beta varies from 3.4 to 5 , while the normalized volume-average beta values have only a $5 \%$ deviation from a mean value of 3.0 with the exception of the case with the lowest triangularity and highest elongation. These combined results imply that $\left\langle\beta_{N}\right\rangle$ is not strictly invariant with respect to large shape variations, as low triangularity apparently degrades $\left\langle\beta_{N}\right\rangle$ limits at sufficiently high elongation as was observed previously [38-40]. However, $\left\langle\beta_{N}\right\rangle$ exhibits significantly less variation as a stability limit parameter than $\beta_{N}$ when either plasma aspect ratio or boundary shape is varied. 


\section{Plasma safety factor scan}

The linear relationship between maximum beta and normalized current can be understood as a natural consequence of the product $q(0) \beta B / I_{P}$ being an invariant of equilibrium and stability at sufficiently high $q(0)$ and aspect ratio [15]. This scaling is well known to break down due to low- $n$ kink instabilities when the magnetic safety factor and shear become sufficiently low and the current limit is reached [41, 42]. For standard aspect ratio tokamaks, this current limit is typically set by the $n=1$ external kink mode and is usually violated when

$q(\hat{\psi}=1) \leq 2[18,43]$. For low aspect ratio, the $q(1)$ limit is less clearly defined [27] at the present time, especially given the potential role of the separatrix [44] in modifying the edge stability and current limits [45].

To better understand the dependence of the current limit on aspect ratio in the present context, plasma current scans with self-driven current fraction ranging from 0 to 0.6 have been performed for three different configurations. Figure 3 a plots $\langle\beta\rangle$ versus $I_{P} / a B_{T 0}$ for these three configurations with geometric parameters $A=1.6, \kappa=2, \delta=0.45$ (squares), $A=1.6$, $\kappa=2.5, \delta=0.6$ (circles), and $A=3.3, \kappa=2, \delta=0.45$ (diamonds). Results from the aspect ratio scan of Figure 1 (crosses) and shape scan of Figure 2a (triangles) are also plotted in this figure. As seen in the figure, the solid line of $\langle\beta\rangle(\%)=3.2 I_{N}(M A / m T)$ represents a good fit to the upper bound of the computed beta limits and demonstrates the nearly linear relationship between maximum volume-average beta and $I_{N}$. Figure $3 \mathrm{~b}$ shows that the $\left\langle\beta_{N}\right\rangle$ limit exhibits a step-wise drop as integer edge $q$-values are passed through from above until the edge $q$ is sufficiently low that the current limit is reached and the equilibrium is unstable even at zero $\beta$. Interestingly, the scan with $A=3.3$ exhibits a current limit at $q(1)=2$ as might be expected for that aspect ratio, whereas the other scans at $A=1.6$ reach the current limit at higher $q(1)$ values which depend on boundary shape. A similar factor of two variation in $q(0.95)$ at the current limit is observed for these scans as shown in Figure 3c. Thus, there appears to be no unique value of $q(1)$ or $q(0.95)$ which determines the current limit independent of aspect ratio. However, if a global safety factor is utilized which accounts for leading order aspect ratio and shape effects, beta limits for currents 
below the current limit can be represented in a more aspect ratio invariant form. This is demonstrated in Figure 3d which plots the $\left\langle\beta_{N}\right\rangle$ limit for all scans performed above versus the cylindrical safety factor $q^{*} \equiv \epsilon\left(1+\kappa^{2}\right) \pi a B_{T 0} / \mu_{0} I_{P}[32,41]$. This figure also shows that $\left\langle\beta_{N}\right\rangle$ degradation begins to occur for $q^{*}$ below 2.0 for all cases treated and no stable cases are found with $q^{*}$ below 1 .

\section{APPLICATION TO NSTX EXPERIMENTAL DATA}

The stability limit scalings discussed above were subject to many constraints which are often not present in actual experiments. For instance, separatrix boundary shapes, H-mode profiles with finite edge pressure gradient and finite edge current density, and local bootstrap current overdrive were not allowed in the numerical studies. Clearly, only detailed stability analysis of a given experimental discharge using measured profiles can determine proximity to a theoretical stability limit. However, provided the experimental profiles and shapes do not differ too dramatically from those used in the scans above, the computed scalings can give guidance as to which discharges are most likely to be exceeding either ballooning or no-wall kink stability limits. In the analysis that follows, NSTX experimental stability limit data is compared to the scalings, and a discharge which violates these scalings is analyzed for ideal MHD stability to test the scaling under more experimentally relevant conditions.

\section{A. Comparison to NSTX stability database}

Recent machine improvements in the NSTX device now allow routine access to the Hmode [46] and its associated low pressure profile peaking $(p(0) /\langle p\rangle \approx 1.8-2.5)$ predicted to be optimal for ideal MHD stability in the ST [32,47]. Figure 4a plots peak NSTX beta values computed with the EFIT equilibrium reconstruction code [48] adapted for NSTX [49] for plasmas with shape parameters spanning $A=1.27-1.5, \kappa=1.5-2.15, \delta=0.25-0.85$ and internal inductance $l_{i}=0.5-1.7$. As seen in the figure, peak beta values have reached $\beta_{T} \leq 35 \%$ with $\beta_{N} \leq 6.5$ and $\left\langle\beta_{N}\right\rangle \leq 4.5$ surpassing previous ST record normalized beta values from the START experiment [27] while achieving comparable $\beta_{T}$ values. Figure $4 \mathrm{~b}$ shows that 
nearly all NSTX discharges have $q(0.95)>3$ at peak beta. However, systematic current limit experiments have not yet been performed to fully test the theoretical limits shown in Figure 3c. Figure 4c shows that the peak $\left\langle\beta_{N}\right\rangle$ in NSTX decreases significantly below 3 as $q^{*}$ drops from 2 to 1 . This trend is consistent with the shaded region shown in Figure $3 \mathrm{~d}$ derived from fits to the stability scan results. In the calculations, the low central $q$ near 1 and weak shear generate largely internal instability eigenfunctions until the current limit is reached. In the experiment, strong core MHD activity (typically in the form of large $m / n=1 / 1$ islands) is often observed at high- $\beta$ in low $q^{*}<2$ discharges. The stability of these predominantly internal modes should be only weakly affected by stabilizing conducting structure outside the plasma, and this tendency may help explain the consistency between the data and simulations exhibited at low $q^{*}$ as shown in Figure 4c. However, many discharges in Figure 4c significantly exceed the upper-bound of this shaded region defined by $\left\langle\beta_{N}\right\rangle=3.5$. The discharges with the highest $\left\langle\beta_{N}\right\rangle$ values $\geq 4$ are indicated by green symbols in Figure 4c. As seen in Figure $4 \mathrm{~d}$, these discharges also have the highest $\left\langle\beta_{N}\right\rangle / l_{i}$ (and $\beta_{N} / l_{i}$ ) ratios obtained thus far in NSTX and meet or slightly exceed the $\beta_{N} / l_{i}$ values from the aspect ratio scan of Figure 1e.

\section{B. Applicability of stability scalings to high-bootstrap-fraction discharges}

The data shown in green in Figures $4 \mathrm{c}$ and $\mathrm{d}$ which exceeds $\left\langle\beta_{N}\right\rangle=3.5$ by as much as $30 \%$ is from an H-mode operational scenario optimized to have very long ELM-free and sawtooth-free periods. These relatively quiescent discharges had plasma parameters $A=1.4$ $1.5, \kappa \approx 2$, average $\delta \approx 0.4$ in a lower single null divertor configuration, $l_{i}=0.6-0.95$, and $q^{*}$ in the range of 2.5-3.0. These discharges reach poloidal beta values above 1.2 (several are measured to be slightly diamagnetic) and EFIT reconstructions and TRANSP current profile diffusion calculations indicate $q(0)>1$ - consistent with the experimental absence of sawteeth and 1/1 islands. However, detailed $q$ profile information is not yet available for NSTX due to a lack of internal magnetic field measurements.

Several time traces for the highest $\left\langle\beta_{N}\right\rangle$ discharge of Figure $4 \mathrm{c}$ are shown in Figure 5 . This 
discharge is particularly relevant to the computational stability scans discussed above, as Figure 5a shows that the bootstrap current fraction as computed by TRANSP [50] reaches $50 \%$ by the end of this discharge with neutral beam injection (NBI) current drive raising the total non-inductive (NI) current fraction to 60-65\%. In this discharge, the density increases steadily and raises the bootstrap current while simultaneously lowering the NBI current drive efficiency and NBI-driven current. The uncertainty in the current drive fractions shown in Figure 5 a arises primarily from uncertainty in the $Z_{\text {eff }}$ profile and the associated uncertainty in the thermal and fast-ion density. Figure 5b plots several normalized beta curves for the same discharge. The three uppermost curves (black) represent $\beta_{N}$, while the three lowermost curves (red) are $\left\langle\beta_{N}\right\rangle$. The solid lines without the circle symbols are experimental partial-kinetic EFIT reconstructions [51] constrained by the diamagnetic flux measurement and loosely by the shape of the electron pressure profile. Full kinetic calculations using the measured $n_{e}, T_{e}$, and $T_{i}$ profiles and fast-ion pressure computed with TRANSP are in good agreement with the EFIT reconstructions for the higher $Z_{\text {eff }}$ values in the range of uncertainty. For the lower $Z_{\text {eff }}$ values, TRANSP predictions of the total stored energy exceed the EFIT reconstructed value by about 10-15\%, and the corresponding normalized beta values are shown by the dashed curves in Figure 5b. For these curves, the agreement between the computed and measured diamagnetic flux is not as good, implying either that the higher $Z_{\text {eff }}$ values are a better match to the experiment or that the fast-ion density in the experiment is smaller than predicted - perhaps due to inaccurate modeling of effects such as edge loss, charge exchange, or anomalous fast-ion diffusion from MHD activity.

Given the aforementioned uncertainty in the equilibrium $q$ profile and the magnitude and shape of the fast-ion pressure profile, it is questionable if accurate marginal stability calculations can be performed to determine the no-wall stability limit for these NSTX discharges. However, it is in principle possible to determine an upper bound on the marginal stability limit given freedom to vary these profiles. Such profile variations have been performed for the discharge of Figure 5, and the $n=1$ kink no-wall marginal stability values computed at three different times are shown by the solid lines with circle symbols in Figure 5b. For these profile variations, a fixed boundary at $\hat{\psi}=0.997$ of the reconstructed lower-single null 
free-boundary solution was used, and the total pressure was treated as the sum of a thermal and fast-ion component using the functional form given by Equation 3. The thermal pressure profile shape used was a fit to the profile computed from TRANSP and included finite edge pressure gradient from the H-mode. The peaking factor of the fast-ion component was systematically varied, and modest broadening of the fast-ion pressure profile was found to increase the stability limit. The current profile was also varied using the functional form of Equation 4 keeping the total plasma current fixed. In this scan, the $q(0)$ value was allowed to vary from 1.4 to 3.5 , and small local bootstrap current overdrive was allowed near the plasma edge. Local ballooning instability was also allowed near the edge - effectively relaxing two of the constraints used in performing the systematic stability scans from Section II. Subsequent variations of the thermal pressure profile showed only small increases in the stable beta once the fast-ion pressure and current profile variations were complete.

With these profile variations and modestly relaxed constraints, Figure 5b shows that $\left\langle\beta_{N}\right\rangle \leq 3.5$ is a reasonable representation of the profile-optimized no-wall beta limit for this discharge. The marginal $\left\langle\beta_{N}\right\rangle$ value of 3.5 is consistent with the upper-bound of the shaded region shown in Figure 3d and exceeds the no-wall limit computed in the more constrained scans of Section II by less than 10\%. These results strongly imply that the experimental $\left\langle\beta_{N}\right\rangle$ shown in Figure 5b exceeds the no-wall limit for several energy confinement times $\left(\tau_{E}=40-50 \mathrm{~ms}\right)$ and many resistive wall times $\left(\tau_{\text {wall }}=5-15 \mathrm{~ms}\right)$. Stabilization of the pressuredriven kink mode from the close fitting conducting plates and beam driven rotation in NSTX $[30,31,51,52]$ is a likely explanation of the violation of the no-wall limit shown in Figures 4c and 5, although shear stabilization of either kink or ballooning modes [53] may also play a role.

\section{SUMMARY}

Systematic ideal no-wall stability calculations scanning plasma aspect ratio, boundary shape, and global safety factor have been performed to test beta limit scalings commonly utilized at higher aspect ratio. An aspect ratio scan with fixed boundary shape and boot- 
strap current fraction finds that no-wall stability limits expressed in terms of a volumeaverage normalized beta parameter exhibit significantly less variation as compared to the conventional normalized beta definition which uses the vacuum toroidal field at the plasma geometric center to normalize the volume-average pressure in the definition of $\beta$. Plasma shape scans at low aspect ratio $(A=1.6)$ also find that the volume-average normalized beta limit exhibits significantly less variation with shape than the toroidal normalized beta, but as for conventional aspect ratio, some degradation in the normalized beta limit is observed for conditions of high elongation and low triangularity. In contrast to this approximate invariance, the maximum no-wall stable $\left\langle\beta_{N}\right\rangle / l_{i}$ ratio increases by at least a factor of 2 at very low aspect ratio, implying that a simple linear scaling between normalized beta and internal inductance does not exist for arbitrary aspect ratio. Plasma current scans at two aspect ratios show that there is no unique edge or near-edge $q$-value which defines the current limit for limiter boundary shapes. However, expressing $\left\langle\beta_{N}\right\rangle$ as a function of $q^{*}$ appears to parameterize both the current limit and $\left\langle\beta_{N}\right\rangle$ degradation at low $q^{*}$ in a more aspect ratio invariant form. Numerically, the optimized no-wall beta limit from all these studies can be summarized as $\left\langle\beta_{N}\right\rangle=3.1 \pm 0.4$ for cylindrical safety factor $q^{*}$ above 1.7. Below this value of $q^{*}$, the no-wall $\left\langle\beta_{N}\right\rangle$ limit decreases rapidly as $q^{*}$ approaches 1 . If any of the constraints of this computational study are relaxed, higher $\left\langle\beta_{N}\right\rangle$ limit values would certainly be expected. For instance, removal of the bootstrap current alignment constraint would in principle allow higher $l_{i}$ solutions which could increase the stable $\left\langle\beta_{N}\right\rangle$ if a $\beta_{N} \propto l_{i}$ scaling is obeyed. Experimentally, NSTX peak $\left\langle\beta_{N}\right\rangle$ values are at or below the profile-optimized no-wall limit for low $q^{*} \leq 2$, but have significantly surpassed this limit for $q^{*}>2$ in some operating scenarios. For the discharges operating above this limit studied thus far, utilizing a diverted boundary shape, H-mode profiles, and allowing for a narrow region of ballooning instability near the edge raises the profile-optimized no-wall $\left\langle\beta_{N}\right\rangle$ limit only $10 \%$ above the values obtained in the systematic scans with limiter boundary shapes and non H-mode profiles. These results imply that some NSTX discharges are operating as much as $30 \%$ above the non-rotating ideal plasma no-wall stability limit. The role of the pressure and current profile shape, wall stabilization, and rotation and rotational shear in allowing operation 
above the profile-optimized no-wall limit will be studied in more detail in future work.

\section{ACKNOWLEDGMENTS}

The main author (JEM) thanks S. C. Jardin, J. Manickam, and A. Glasser for their help with the equilibrium and stability codes used in this work. This research was supported by the United States Department of Energy under contract numbers DE-AC02-76CH03037 (PPPL) and DE-AC05-00R22725 (ORNL), and grant numbers DE-FG02-99ER54524 (CU) and DE-FG02-99ER54523 (JHU). 
[1] M. Kikuchi, Plasma Phys. and Contr. Fus. 35, B39 (1993).

[2] R. J. Goldston, S. H. Batha, R. H. Bulmer, D. N. Hill, A. W. Hyatt, S. C. Jardin, F. M. Levinton, S. M. Kaye, C. E. Kessel, E. A. Lazarus, et al., Plasma Phys. and Contr. Fus. 36, B213 (1994).

[3] T. S. Taylor, H. S. John, A. D. Turnbull, Y. R. Lin-Liu, K. H. Burrell, V. Chan, M. S. Chu, J. R. Ferron, L. L. Lao, R. J. L. Haye, et al., Plasma Phys. and Contr. Fus. 36, B229 (1994).

[4] S. Jardin, C. Kessel, C. Bathke, D. Ehst, T. Mau, F. Najmabadi, and T. Petrie, Fus. Engin. and Design 38, 27 (1997).

[5] C. Kessel, T. Mau, S. Jardin, and F. Najmabadi, Tech. Rep. PPPL-3573, Princeton Plasma Physics Laboratory (2001), Plasma profile and shape optimization for the advanced tokamak power plant, ARIES-AT, Submitted for publication to Fus. Eng. Design.

[6] S. Hirshman, D. Spong, J. Whitson, V. Lynch, D. Batchelor, B. Carreras, and J. Rome, Phys. Rev. Lett. 80, 528 (1998).

[7] M. C. Zarnstorff, L. A. Berry, A. Brooks, E. Fredrickson, G.-Y. Fu, S. Hirshman, S. Hudson, L.-P. Ku, E. Lazarus, D. Mikkelsen, et al., Plasma Phys. and Contr. Fus. 43, 237 (2001).

[8] Y.-K. M. Peng and D. J. Strickler, Nucl. Fus. 26, 769 (1986).

[9] R. D. Stambaugh, V. S. Chan, R. L. Miller, and M. J. Schaffer, Fus. Technol. 33, 1 (1998).

[10] S. C. Jardin, C. E. Kessel, J. Menard, T. K. Mau, F. Najmabadi, R. L. Miller, A. D. Turnbull, and R. D. Stambaugh, Fus. Engin. and Design 65, 165 (2003).

[11] R. J. Bickerton, J. W. Connor, and J. B. Taylor, Nature Phys. Sci. 229, 110 (1971).

[12] A. Sykes, M. Turner, and S. Patel, in Proceedings of the 11th European Conference on Controlled Fusion and Plasma Physics, Aachen, Germany, 1983, edited by S. Methfessel, European Physical Society (Petit-Lancy, Switzerland, 1983), vol. 7D, Part II, p. 363.

[13] F. Troyon, R. Gruber, H. Saurenmann, S. Semenzato, and S. Succi, Plasma Phys. and Contr. Fus. 26, 209 (1984).

[14] J. A. Wesson and A. Sykes, Nucl. Fus. 25, 85 (1985). 
[15] J. J. Ramos, Phys. Rev. A 42, 1021 (1990).

[16] F. Troyon and R. Gruber, Phys. Lett. A 110, 29 (1985).

[17] R. E. Bell, N. Asakura, S. Bernabei, M. S. Chance, P.-A. Duperrex, R. J. Fonck, G. M. Gammel, G. Greene, R. E. Hatcher, A. Holland, et al., Phys. Fluids B 2, 1271 (1990).

[18] E. Strait, Phys. Plasmas 1, 1415 (1994).

[19] E. J. Strait, T. S. Taylor, A. D. Turnbull, J. R. Ferron, L. L. Lao, B. Rice, O. Sauter, S. J. Thompson, and D. Wroblewski, Phys. Rev. Lett. 74, 2483 (1995).

[20] M. R. Wade, T. C. Luce, P. A. Politzer, J. R. Ferron, S. L. Allen, M. E. Austin, D. R. Baker, B. Bray, D. P. Brennen, K. H. Burrell, et al., Phys. Plasmas 8, 2208 (2001).

[21] T. Fujita, S. Ide, Y. Kamada, T. Suzuki, T. Oikawa, S. Takeji, Y. Sakamoto, Y. Koide, A. Isayama, T. Hatae, et al., Phys. Rev. Lett. 87, 085001 (2001).

[22] J. Hobirk, R. C. Wolf, O. Gruber, A. Gude, S. Gunter, B. Kurzan, M. Maraschek, P. J. McCarthy, H. Meister, A. G. Peeters, et al., Phys. Rev. Lett. 87, 085002 (2001).

[23] A. M. Garofalo, E. J. Strait, L. C. Johnson, R. J. L. Haye, E. A. Lazarus, G. A. Navratil, M. Okabayashi, J. T. Scoville, T. S. Taylor, and A. D. Turnbull, Phys. Rev. Lett. 89, 235001 (2002).

[24] A. Sykes, E. Delbosco, R. Colchin, G. Cunningham, R. Duck, T. Edlington, D. Goodall, M. Gryaznevich, J. Holt, J. Hugill, et al., Nucl. Fus. 32, 694 (1992).

[25] D. A. Gates, R. Akers, L. Appel, P. G. Carolan, N. Conway, J. Dowling, M. Gryaznevich, T. Hender, O. J. Kwon, R. Martin, et al., Phys. Plasmas 5, 1775 (1998).

[26] M. Gryaznevich, R. Akers, P. G. Carolan, N. J. Conway, D. Gates, A. R. Field, T. C. Hender, I. Jenkins, R. Martin, M. P. S. Nightingale, et al., Phys. Rev. Lett. 80, 3972 (1998).

[27] A. Sykes, the START Team, the NBI Team, the MAST Team, and the Theory Team, Nucl. Fus. 39, 1271 (1999).

[28] A. Sykes, R. J. Akers, L. C. Appel, E. R. Arends, P. G. Carolan, N. J. Conway, G. F. Counsell, G. Cunningham, A. Dnestrovskij, Y. N. Dnestrovskij, et al., Nucl. Fus. 41, 1423 (2001).

[29] M. Ono, S. Kaye, Y.-K. Peng, G. Barnes, W. Blanchard, M. Carter, J. Chrzanowski, L. Dudek, R. Ewig, D. Gates, et al., Nucl. Fus. 40, 557 (2000). 
[30] J. E. Menard, M. G. Bell, R. E. Bell, E. D. Fredrickson, D. A. Gates, S. M. Kaye, B. P. LeBlanc, R. Maingi, D. Mueller, S. A. Sabbagh, et al., Nucl. Fus. 43, 330 (2003).

[31] D. Gates and the NSTX National Research Team, Phys. Plasmas 10, 1659 (2003).

[32] J. E. Menard, S. C. Jardin, S. M. Kaye, C. E. Kessel, and J. Manickam, Nucl. Fus. 37, 595 (1997).

[33] A. Glasser and M. Chance, Bull. Am. Phys. Soc. 42, 1848 (1997).

[34] T. Ozeki, M. Azumi, S. Tokuda, and S. Ishida, Nucl. Fus. 33, 1025 (1993).

[35] M. W. Phillips, M. C. Zarnstorff, J. Manickam, and F. M. L. M. H. Hughes, Phys. Plasmas 3, 1673 (1996).

[36] A. Bondeson, M. Benda, M. Persson, and M. S. Chu, Nucl. Fus. 37, 1419 (1997).

[37] A. D. Turnbull, T. S. Taylor, M. S. Chu, R. L. Miller, and Y. R. Lin-Liu, Nucl. Fus. 38, 1467 (1998).

[38] Y. R. Lin-Liu, A. D. Turnbull, M. S. Chu, J. R. Ferron, R. L. Miller, and T. S. Taylor, Phys. Plasmas 6, 3934 (1999).

[39] K. Yamazaki, T. Amano, H. Naitou, Y. Hamada, and M. Azumi, Nucl. Fus. 25, 1543 (1985).

[40] M. W. Phillips, A. M. M. Todd, M. H. Hughs, J. Manickam, J. L. Johnson, and R. R. Parker, Nucl. Fus. 28, 1499 (1988).

[41] D. A. D’Ippolito, J. P. Freidberg, J. P. Goedbloed, and J. Ram, Phys. Fluids 21, 1600 (1978).

[42] A. D. Turnbull, A. Roy, O. Sauter, and F. S. Troyon, Nucl. Fus. 28, 1379 (1988).

[43] F. Hofmann, O. Sauter, H. Reimerdes, I. Furno, and A. Pochelon, Phys. Rev. Lett. 81, 2918 (1998).

[44] L. Degtyarev, A. Martynov, S. Medvedev, F. Troyon, L. Villard, and R. Gruber, Comput. Phys. Commun. 103, 10 (1997).

[45] S. Y. Medvedev, T. C. Hender, O. Sauter, and L. Villard, in 28th EPS Conference on Controlled Fusion and Plasma Physics (European Physical Society, Funchal, 2001), vol. 25A, p. 21.

[46] R. Maingi, M. G. Bell, R. E. Bell, C. E. Bush, E. D. Fredrickson2, D. A. Gates, S. M. Kaye, H. W. Kugel, B. P. LeBlanc, J. E. Menard, et al., Phys. Rev. Lett. 88, 035003 (2002). 
[47] F. Paoletti, S. Sabbagh, J. Manickam, J. Menard, R. Akers, D. Gates, S. Kaye, and L. Lao, Nucl. Fus. 42, 418 (2002).

[48] L. L. Lao, H. St. John, R. D. Stambaugh, A. G. Kellman, and W. Pfeiffer, Nucl. Fus. 25, 1611 (1985).

[49] S. A. Sabbagh, S. M. Kaye, J. Menard, M. Bell, R. Bell, M. Bitter, E. Fredrickson, D. Gates, A. H. Glasser, L. L. Lao, et al., Nucl. Fus. 41, 1601 (2001).

[50] R. J. Goldston, D. C. McCune, and H. H. Towner, J. Comput. Phys. 43, 61 (1981).

[51] S. A. Sabbagh, R. E. Bell, M. G. Bell, J. Bialek, A. H. Glasser, B. LeBlanc, J. E. Menard, F. Paoletti, D. Stutman, E. Fredrickson, et al., Phys. Plasmas 9, 2085 (2002).

[52] S. A. Sabbagh, J. M. Bialek, R. E. Bell, A. H. Glasser, B. P. LeBlanc, J. E. Menard, F. Paoletti, M. G. Bell, R. Fitzpatrick, E. D. Fredrickson, et al., The resistive wall mode and feedback control physics design in NSTX (2003), submitted to Nuclear Fusion.

[53] F. Waelbroeck and L. Chen, Phys. Fluids B 3, 601 (1991). 


\section{Figure Captions}

Figure 1 - (a) Toroidal and volume-average beta, (b) normalized toroidal and volumeaverage beta values, (c) pressure profile peaking factor, (d) central safety factor and inverse internal inductance, and (e) normalized beta values divided by internal inductance versus aspect ratio for $50 \%$ self-driven current fraction plasmas with $\kappa=2.0$ and $\delta=0.45$.

Figure 2 - (a) Marginally stable $\beta_{T}(\%)$ and (b) normalized beta values as a function of triangularity and elongation at 50\% self-driven current fraction for aspect ratio $A=1.6$.

Figure 3 - (a) Marginally stable volume-average beta versus $I_{P} / a B_{T 0}$ for all stability scans performed. Volume-average normalized beta versus (b) edge safety factor, and (c) safety factor at 95\% normalized poloidal flux for the plasma current scans. (d) Volumeaverage normalized beta versus cylindrical safety factor $q^{*}$ for all stability scans performed.

Figure 4 - (a) $\beta_{T}$ (black) and $\langle\beta\rangle$ (red) at maximum stored energy for NSTX neutral beam heated discharges plotted versus normalized current, (b) $\left\langle\beta_{N}\right\rangle$ versus safety factor at surface of 95\% normalized poloidal flux, (c) $\left\langle\beta_{N}\right\rangle$ versus cylindrical safety factor, and (d) $\beta_{N} / l_{i}$ (black) and $\left\langle\beta_{N}\right\rangle / l_{i}$ (red) versus inverse aspect ratio. Constant normalized beta lines in (a) are shown in blue. Black and red curves in (d) are from Figure 1e.

Figure 5 - (a) Non-inductive (NI) and bootstrap (BS) current fractions, and (b) normalized $\beta$ values versus time for discharge 109070. 


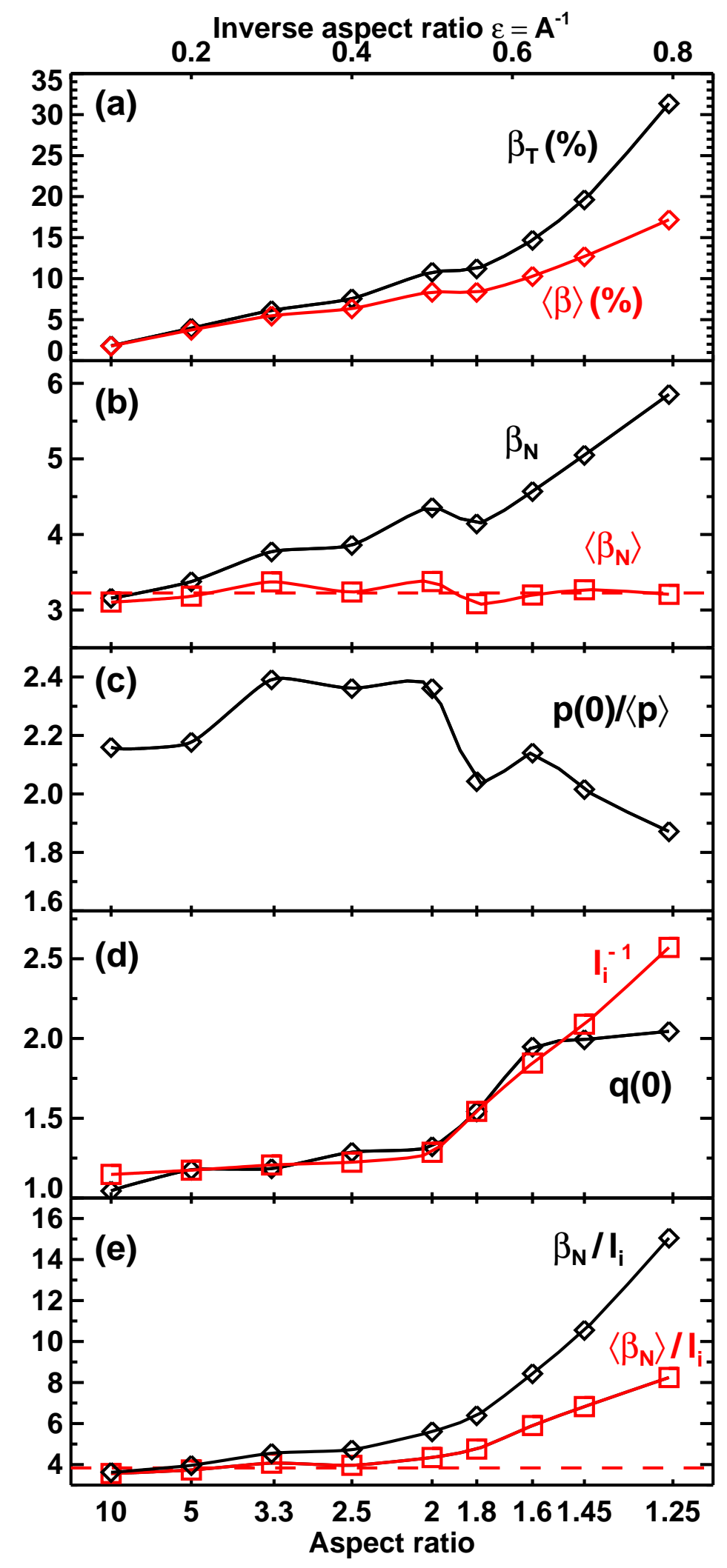

FIG. 1: 

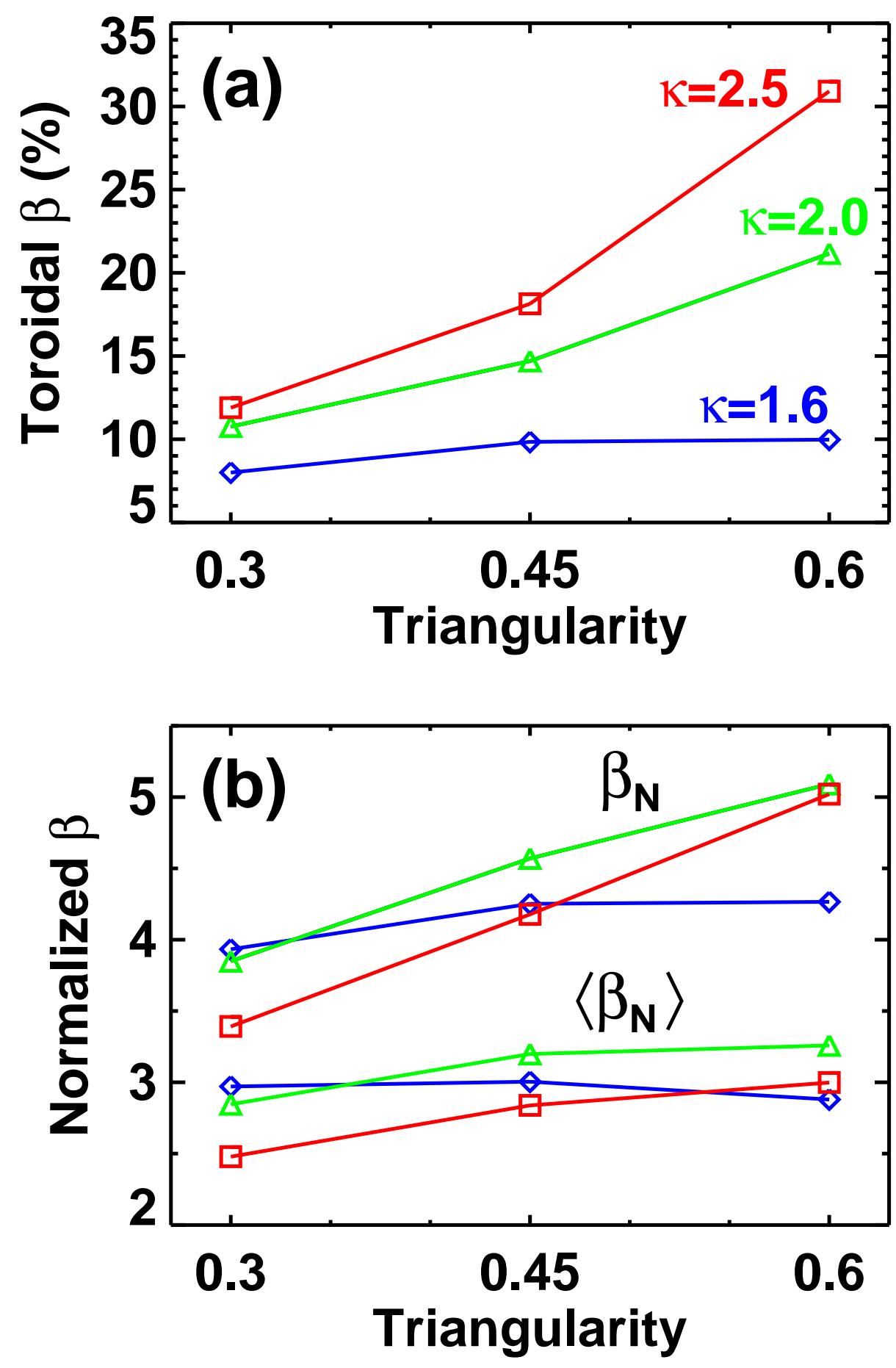

FIG. 2: 

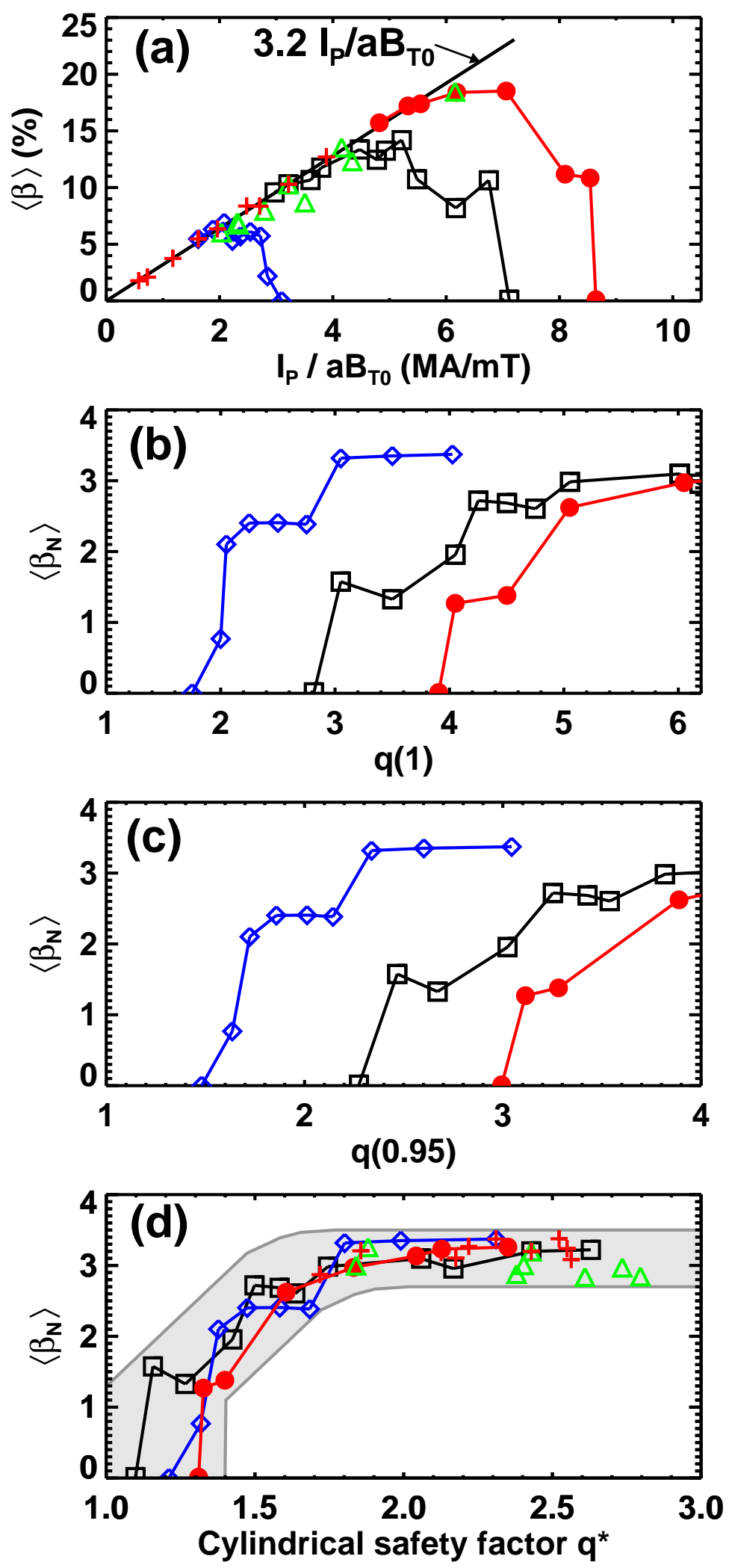

FIG. 3: 

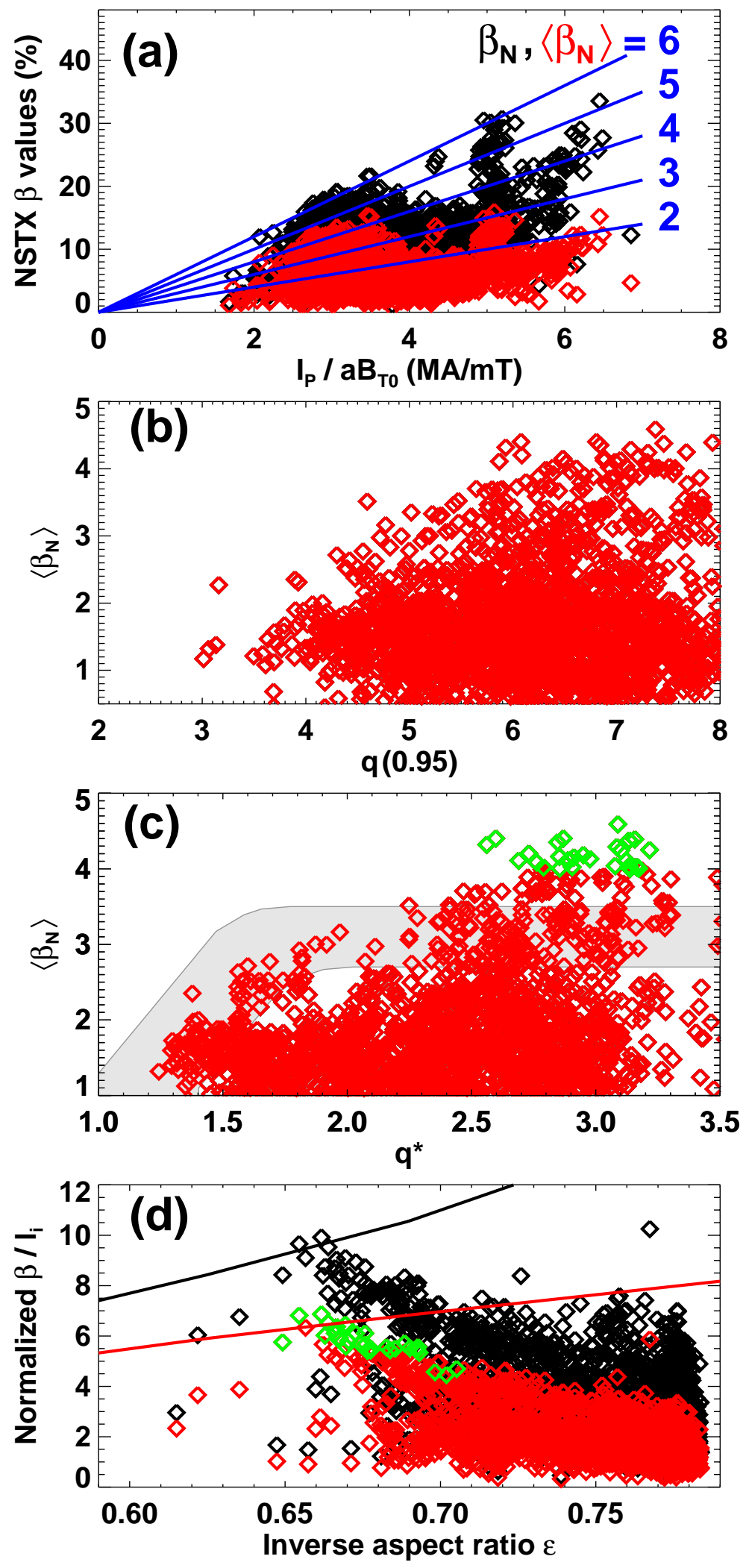

FIG. 4: 

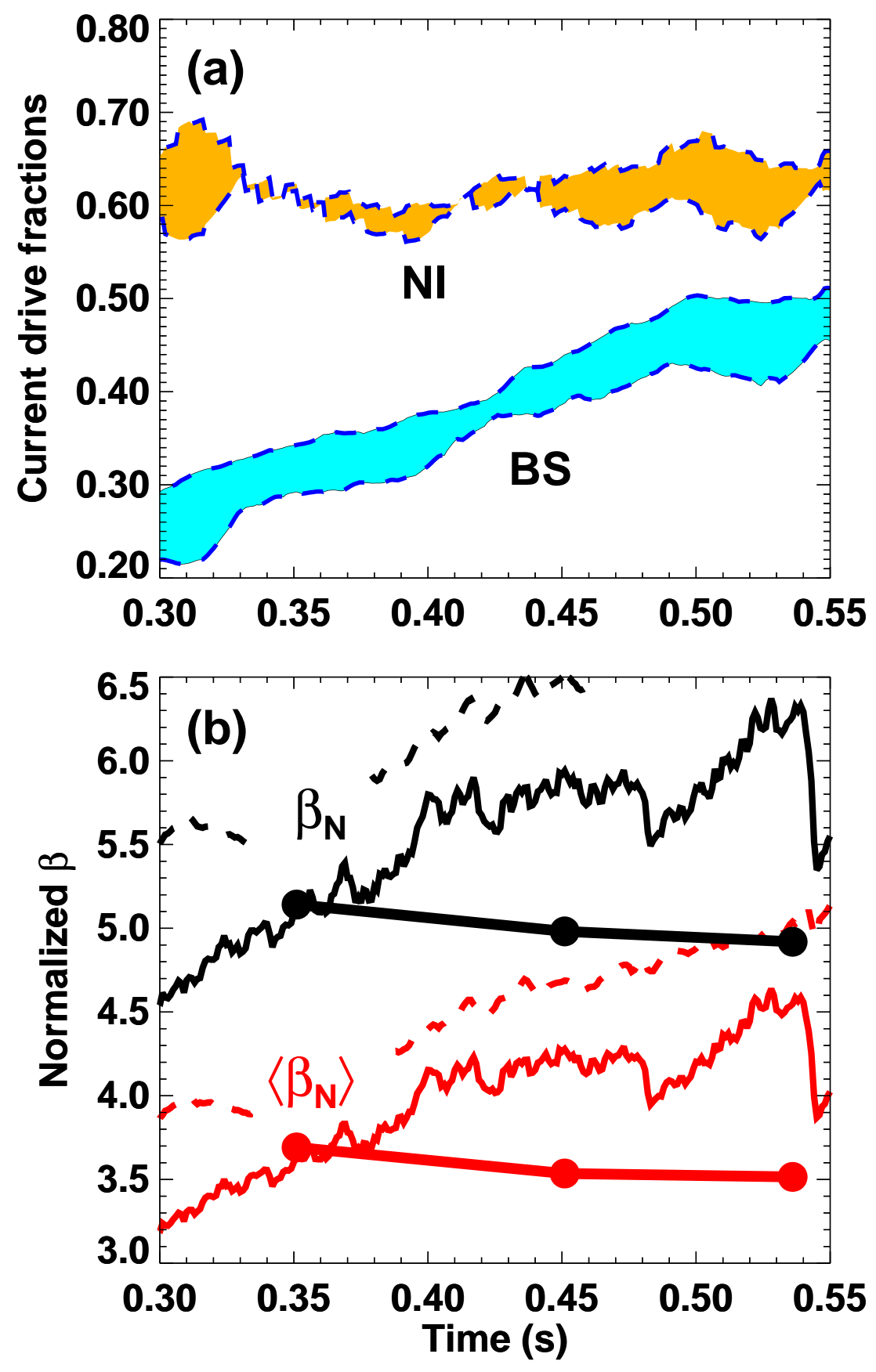

FIG. 5: 


\section{External Distribution}

Plasma Research Laboratory, Australian National University, Australia

Professor I.R. Jones, Flinders University, Australia

Professor João Canalle, Instituto de Fisica DEQ/IF - UERJ, Brazil

Mr. Gerson O. Ludwig, Instituto Nacional de Pesquisas, Brazil

Dr. P.H. Sakanaka, Instituto Fisica, Brazil

The Librarian, Culham Laboratory, England

Mrs. S.A. Hutchinson, JET Library, England

Professor M.N. Bussac, Ecole Polytechnique, France

Librarian, Max-Planck-Institut für Plasmaphysik, Germany

Jolan Moldvai, Reports Library, Hungarian Academy of Sciences, Central Research Institute for Physics, Hungary

Dr. P. Kaw, Institute for Plasma Research, India

Ms. P.J. Pathak, Librarian, Institute for Plasma Research, India

Ms. Clelia De Palo, Associazione EURATOM-ENEA, Italy

Dr. G. Grosso, Instituto di Fisica del Plasma, Italy

Librarian, Naka Fusion Research Establishment, JAERI, Japan

Library, Laboratory for Complex Energy Processes, Institute for Advanced Study, Kyoto University, Japan

Research Information Center, National Institute for Fusion Science, Japan

Dr. O. Mitarai, Kyushu Tokai University, Japan

Dr. Jiangang Li, Institute of Plasma Physics, Chinese Academy of Sciences, People's Republic of China

Professor Yuping Huo, School of Physical Science and Technology, People's Republic of China

Library, Academia Sinica, Institute of Plasma Physics, People's Republic of China

Librarian, Institute of Physics, Chinese Academy of Sciences, People's Republic of China

Dr. S. Mirnov, TRINITI, Troitsk, Russian Federation, Russia

Dr. V.S. Strelkov, Kurchatov Institute, Russian Federation, Russia

Professor Peter Lukac, Katedra Fyziky Plazmy MFF UK, Mlynska dolina F-2, Komenskeho Univerzita, SK-842 15 Bratislava, Slovakia

Dr. G.S. Lee, Korea Basic Science Institute, South Korea

Institute for Plasma Research, University of Maryland, USA

Librarian, Fusion Energy Division, Oak Ridge National Laboratory, USA

Librarian, Institute of Fusion Studies, University of Texas, USA

Librarian, Magnetic Fusion Program, Lawrence Livermore National Laboratory, USA

Library, General Atomics, USA

Plasma Physics Group, Fusion Energy Research Program, University of California at San Diego, USA

Plasma Physics Library, Columbia University, USA

Alkesh Punjabi, Center for Fusion Research and Training, Hampton University, USA

Dr. W.M. Stacey, Fusion Research Center, Georgia Institute of Technology, USA

Dr. John Willis, U.S. Department of Energy, Office of Fusion Energy Sciences, USA

Mr. Paul H. Wright, Indianapolis, Indiana, USA 
The Princeton Plasma Physics Laboratory is operated by Princeton University under contract with the U.S. Department of Energy.

\author{
Information Services \\ Princeton Plasma Physics Laboratory \\ P.O. Box 451 \\ Princeton, NJ 08543
}

Phone: 609-243-2750

Fax: 609-243-2751

e-mail: pppl_info@pppl.gov

Internet Address: http://www.pppl.gov 